PROCEEDINGS OF THE

AMERICAN MATHEMATICAL SOCIETY

Volume 138, Number 12, December 2010, Pages 4321-4329

S 0002-9939(2010)10433-1

Article electronically published on May 24, 2010

\title{
THE DEFICIENCY OF A COHOMOLOGY CLASS
}

\author{
C. A. MORALES
}

(Communicated by Daniel Ruberman)

\begin{abstract}
We define the deficiency of a cohomology class $u$ with respect to a vector field as the set of limit points in the ambient manifold of long almost closed orbits representing homology classes on which $u$ is nonpositive. We prove that, up to infinite cyclic coverings, the sole vector fields on closed manifolds exhibiting nonzero cohomology classes with finite deficiency are the gradient-like ones. We also prove that if the manifold is not a sphere, every singularity is hyperbolic and there is a closed transverse submanifold intersecting all regular orbits, then there is also a nonzero cohomology class with finite deficiency.
\end{abstract}

\section{INTRODUCTION}

Limits of long almost closed orbits for vector fields are dynamical objects widely studied in the literature. For instance, Fried used them to defined homology directions as the set of limit points in the normalized homology of homology classes corresponding to long almost closed orbits. It was proved that there is a cohomology class which is positive at every homology direction if and only if the flow is suspended, i.e., exhibits a closed transverse submanifold intersecting all orbits. Moreover, there is a cohomology class which is positive at every homology direction if and only if the vector field is polar up to infinite cyclic coverings; i.e., there is an infinite cyclic covering such that every positive (resp. negative) orbit of the lifted flow goes to $\infty$ (resp. $-\infty$ ). See [5] for details. Afterward, Fried himself studied the zeta function for Axiom A flows using homology directions 6, while Andrade proved the generic stability of the homology directions under small $C^{0}$ perturbations [1. The case when a cohomology class is nonnegative but zero at some homology directions for suspended pseudo-Anosov flows was considered first by Mosher 9 (see also [10). He also studied the dual $C$ under Poincaré pairing of the homology directions for pseudo-Anosov flows $X$ on closed 3-manifolds [12. It was proved in the quasigeodesic case that $C \cap \chi^{-1}(-1)$ is a face of the unit ball of the Thurston norm on the second real homology group ( $\chi$ stands for the Euler class of the normal bundle of $X$ ). Basener described the homology directions in [3] using his previous result 2, while Collier and Sharp proved that they are equidistributed for transitive Anosov flows 4 .

Received by the editors October 28, 2009 and, in revised form, February 1, 2010

2000 Mathematics Subject Classification. Primary 37C10; Secondary 37C50.

Key words and phrases. Cohomology class, deficiency, vector field.

This research was partially supported by CNPq, FAPERJ and PRONEX-Brazil.

(C)2010 American Mathematical Society Reverts to public domain 28 years from publication 
In this paper we further explore limits of long almost closed orbits but directly in the ambient manifold rather than in the normalized homology. More precisely, we define the deficiency of an integer cohomology class $u$ with respect to a vector field $X$ on a closed manifold $M$ as the set of limit points in $M$ of long almost closed orbits corresponding to homology classes on which $u$ is nonpositive. At first glance we notice that the deficiency is a compact invariant set which is empty precisely when the cohomology class is positive at every homology direction. In particular, we have the following three equivalent properties:

- There is a cohomology class with empty deficiency.

- The vector field is polar up to infinite cyclic coverings.

- There is a closed transverse submanifold intersecting all orbits.

(This justifies the name deficiency: the smaller it is, the more likely the flow will be suspended.) These equivalences suggest that we should analyze the case when there is a nonzero cohomology class with finite deficiency. In such a case we prove with the aid of 5 that the vector field is gradient-like up to infinite cyclic coverings; that is, there is an infinite cyclic covering such that every positive (resp. negative) orbit of the lifted flow goes to $\infty$ (resp. $-\infty$ ) or a singularity. However, the converse of this assertion is false as one can easily construct vector fields with infinitely many singularities on the 2-torus which nevertheless are gradient-like up to infinite cyclic coverings. We also prove that if $M$ is not homeomorphic to a sphere, every singularity of $X$ is hyperbolic and there is a closed transverse submanifold intersecting all regular orbits, then there is also a nonzero cohomology class with finite deficiency. The converse of this last assertion is false too since the Cherry flow in the 2-torus 13 is a counterexample. Let us state our results in a precise way.

Hereafter $M$ denotes a closed manifold (i.e., a compact connected boundaryless manifold) equipped with distance $d$ induced by a Riemannian metric. Sometimes we say that $M$ is an $n$-manifold to say that $M$ has dimension $n \in \mathbb{N}$. Denote by $H_{1}(M, \mathbb{Z})$ and $H^{1}(M, \mathbb{Z})$ the first integer homology and cohomology groups of $M$ respectively. Recall the well known identity $H^{1}(M, \mathbb{Z})=\operatorname{Hom}\left(H_{1}(M, \mathbb{Z}), \mathbb{Z}\right)$ where $\operatorname{Hom}(\cdot, \cdot)$ stands for the homomorphism functor. Given $x, y \in M$ we denote by $\gamma_{x, y}$ the minimal geodesic from $x$ to $y$ which is well defined for $d(x, y)$ small enough. Denote by $\alpha * \beta$ the product of two curves $\alpha$ and $\beta$ for which the initial point of $\beta$ and the endpoint of $\alpha$ coincide. The homology class of a closed path $c$ in $M$ will be denoted by $[c]$.

Let $X$ be a vector field in $M$ and denote by $X_{t}$ its corresponding flow. If $A \subset M$ and $B \subset \mathbb{R}$ we define $X_{B}(A)=\left\{X_{t}(x):(x, t) \in A \times B\right\}$ and write $X_{B}(x)$ instead of $X_{B}(\{x\})$ when $A$ reduces to the singleton $\{x\}$. Notice that if $B=[a, b]$ is an interval, then $X_{[a, b]}(x)$ is a curve from $X_{a}(x)$ to $X_{b}(x)$. In particular, the closed path $\gamma_{X_{b}(x), X_{a}(x)} * X_{[a, b]}(x)$ as well as its corresponding homology class $\left[\gamma_{X_{b}(x), X_{a}(x)} * X_{[a, b]}(x)\right] \in H_{1}(M, \mathbb{Z})$ are well defined whenever $d\left(X_{b}(x), X_{a}(x)\right)$ is small enough. We call $X_{\mathbb{R}}(x)$ the orbit of $x \in M$. A singularity is a point $\sigma$ whose orbit reduces to the singleton $\{\sigma\}$. All vector fields in this paper will be of class $C^{1}$.

Definition 1.1. The deficiency of a cohomology class $u \in H^{1}(M, \mathbb{Z})$ with respect to $X$ is the set $\operatorname{def}(u)$ consisting of those $x \in M$ for which there are sequences $x_{k} \in M, t_{k} \in \mathbb{R}^{+}$and $0 \leq \hat{t}_{k} \leq t_{k}$ such that $t_{k} \rightarrow \infty, d\left(X_{t_{k}}\left(x_{k}\right), x_{k}\right) \rightarrow 0$, $X_{\hat{t}_{k}}\left(x_{k}\right) \rightarrow x$ and $u\left(\left[\gamma_{X_{t_{k}}\left(x_{k}\right), x_{k}} * X_{\left[0, t_{k}\right]}\left(x_{k}\right)\right]\right) \leq 0$ for all $k$. 
Our first result will present some consequences of the existence of nonzero cohomology classes with finite deficiency. For this, recall that a regular covering of $M$ is infinite cyclic if its group of deck transformations is $\mathbb{Z}$; see [15]. As is well known, all such coverings admit a two-point compactification consisting of adjoining two ends $\{\infty,-\infty\}$ with the following property: If $g$ is a preferred generator of the deck transformations, then $g^{n}(\tilde{x}) \rightarrow \infty$ or $-\infty$ depending on whether $n \rightarrow \infty$ or $-\infty$ for all $\tilde{x}$ in the total space. With these definitions we shall prove the following.

Theorem 1.2. A vector field on a closed manifold exhibiting nonzero cohomology classes with finite deficiency is gradient-like up to infinite cyclic covering. [5].

The proof will be obtained by adapting the proof of Theorem D on page 357 in

Now we give sufficient conditions for existence of nonzero cohomology classes with finite deficiency. Denote by $S^{n}$ the $n$-dimensional sphere, $n \geq 1$. We shall write $M \neq S^{n}$ to mean that $M$ is not homeomorphic to $S^{n}$. A singularity $\sigma$ is hyperbolic if its eigenvalues have nonzero real part and a regular orbit is an orbit which is not a singularity. Of course we must exclude the sphere $S^{n}$ in the statement below.

Theorem 1.3. Let $X$ be a vector field all of whose singularities are hyperbolic on a closed $n$-manifold $\neq S^{n}, n \geq 2$. If $X$ exhibits a closed transverse submanifold intersecting all regular orbits, then it also exhibits a nonzero cohomology class with finite deficiency.

In particular we have the following corollary. For simplicity let us say that a vector field on a closed manifold is singular-suspended if it exhibits a closed transverse submanifold intersecting all regular orbits. Clearly a suspended vector field is singular-suspended but not conversely.

Corollary 1.1. Every singular-suspended vector field all of whose singularities are hyperbolic on a closed manifold $\neq S^{n}, n \geq 2$, is gradient-like up to infinite cyclic coverings.

The converse of this corollary is false; namely, there are vector fields all of whose singularities are hyperbolic on the 2-torus which are gradient-like up to infinite cyclic coverings but not singular-suspended (e.g. the Cherry flow [13).

\section{Proofs AND EXAMPleS}

Hereafter $X$ will denote a vector field on a closed Riemannian manifold $M$. Recall that a subset $\Lambda \subset M$ is invariant if $X_{t}(\Lambda)=\Lambda$ for all $t \in \mathbb{R}$.

Lemma 2.1. The deficiency is a compact invariant set.

Proof. The compactness is clear so we only have to prove the invariance. Take a cohomology class $u$ and $x \in \operatorname{def}(u)$. Hence there are sequences $x_{k} \in M, t_{k} \in \mathbb{R}^{+}$ and $0 \leq \hat{t}_{k} \leq t_{k}$ such that $t_{k} \rightarrow \infty, d\left(X_{t_{k}}\left(x_{k}\right), x_{k}\right) \rightarrow 0, X_{\hat{t}_{k}}\left(x_{k}\right) \rightarrow x$ and $u\left(\left[\gamma_{X_{t_{k}}\left(x_{k}\right), x_{k}} * X_{\left[0, t_{k}\right]}\left(x_{k}\right)\right]\right) \leq 0$ for all $k$. Now take $t \in \mathbb{R}$ and define $x_{k}^{t}=$ $X_{t}\left(x_{k}\right)$. Then, $d\left(X_{t_{k}}\left(x_{k}^{t}\right), x_{k}^{t}\right) \rightarrow 0$ and $X_{\hat{t}_{k}}\left(x_{k}^{t}\right) \rightarrow X_{t}(x)$ since $X_{t}$ is continuous. Next we observe that $X_{\left[0, t_{k}\right]}\left(x_{k}^{t}\right)=X_{t}\left(X_{\left[0, t_{k}\right]}\left(x_{k}\right)\right)$. Moreover, the curves $X_{t}\left(\gamma_{X_{t_{k}}\left(x_{k}\right), x_{k}}\right)$ and $\gamma_{X_{t_{k}}\left(x_{k}^{t}\right), x_{k}^{t}}$ have the same endpoints and belong to a common ball, so they are homotopic with endpoints fixed. Consequently, the 1-cycles 
$\gamma_{X_{t_{k}}\left(x_{k}^{t}\right), x_{k}^{t}} * X_{\left[0, t_{k}\right]}\left(x_{k}^{t}\right)$ and $\gamma_{X_{t_{k}}\left(x_{k}\right), x_{k}} * X_{\left[0, t_{k}\right]}\left(x_{k}\right)$ are homologous, so $\left[\gamma_{X_{t_{k}}\left(x_{k}^{t}\right), x_{k}^{t} *} *\right.$ $\left.X_{\left[0, t_{k}\right]}\left(x_{k}^{t}\right)\right]=\left[\gamma_{X_{t_{k}}\left(x_{k}\right), x_{k}} * X_{\left[0, t_{k}\right]}\left(x_{k}\right)\right]$ for all $k$. From this and the fact that the time $t$-map $X_{t}$ is homotopic to the identity we get

$$
u\left(\left[\gamma_{X_{t_{k}}\left(x_{k}^{t}\right), x_{k}^{t}} * X_{\left[0, t_{k}\right]}\left(x_{k}^{t}\right)\right]\right)=u\left(\left[\gamma_{X_{t_{k}}\left(x_{k}\right), x_{k}} * X_{\left[0, t_{k}\right]}\left(x_{k}\right)\right]\right) \leq 0, \quad \forall k .
$$

Therefore $X_{t}(x) \in \operatorname{def}(u)$, which proves that $\operatorname{def}(u)$ is invariant.

Proof of Theorem 1.2. We proceed as in the proof of Theorem D in [5]. Let $X$ be a vector field on a closed manifold $M$ exhibiting a nonzero cohomology class $u$ with finite deficiency. Denote by $\operatorname{Sing}(X)$ the set of singularities of $X$.

We can associate the homomorphism $\hat{u}: \pi_{1}(M) \rightarrow \mathbb{Z}$ defined by $\hat{u}\left([c]_{x_{0}}\right)=u([c])$, where $[\cdot]_{x_{0}}$ denotes the homotopy class with respect to a fixed base point $x_{0}$ for the fundamental group $\pi_{1}(M)$. For such a homomorphism we can consider the Galois covering $p: \tilde{M} \rightarrow M$ associated to $\operatorname{Ker}(\hat{u})$, namely, $p_{\#}\left(\pi_{1}(\tilde{M})\right)=\pi_{1}(M)$ where the fundamental group $\pi_{1}(\tilde{M})$ has base point $\tilde{x}_{0} \in p^{-1}\left(x_{0}\right)$. Here $p_{\#}$ stands for the induced map. Since $u \neq 0$ we have that this covering is infinite cyclic.

Let us first prove that every positive orbit of the lift $\tilde{X}$ of $X$ to $\tilde{M}$ goes to $\infty$, $-\infty$ or a singularity. Suppose by contradiction that there is $\tilde{x} \in \tilde{M}$ whose positive orbit under $\tilde{X}$ goes to neither $\infty$ nor $-\infty$ nor a singularity. Then, there is a real number sequence $\tilde{t}_{k}$ with $\tilde{t}_{k+1}-\tilde{t}_{k} \rightarrow \infty$ such that $\tilde{X}_{\tilde{t}_{k}}(\tilde{x}) \rightarrow \tilde{y}$ for some regular point $\tilde{y} \in \tilde{M} \backslash\{ \pm \infty\}$ of $\tilde{X}$. Defining $t_{k}=\tilde{t}_{k+1}-\tilde{t}_{k}$ and $x_{k}=p\left(\tilde{X}_{\tilde{t}_{k}}(\tilde{x})\right)$ we get $t_{k} \rightarrow \infty$ and

$$
d\left(X_{t_{k}}\left(x_{k}\right), x_{k}\right)=d\left(p\left(\tilde{X}_{\tilde{t}_{k+1}}(\tilde{x})\right), p\left(\tilde{X}_{\tilde{t}_{k}}(\tilde{x})\right)\right) \rightarrow d(p(\tilde{y}), p(\tilde{y}))=0 .
$$

In particular, we can consider the closed paths $\gamma_{X_{t_{k}}\left(x_{k}\right), x_{k}} * X_{\left[0, t_{k}\right]}\left(x_{k}\right)$.

For all $k$ large, take a path $\tilde{\beta}_{k}$ from the base point $\tilde{x}_{0}$ to $\tilde{X}_{\tilde{t}_{k}}(\tilde{x})$ and set $\beta_{k}=p \circ \tilde{\beta}_{k}$. Notice that

$$
X_{\left[0, t_{k}\right]}\left(x_{k}\right)=X_{\left[0, t_{k}\right]}\left(p\left(\tilde{X}_{\tilde{t}_{k}}(\tilde{x})\right)\right)=p\left(\tilde{X}_{\left[\tilde{t}_{k}, \tilde{t}_{k+1}\right]}(\tilde{x})\right) .
$$

Denote by $\tilde{\gamma}_{\tilde{a}, \tilde{b}}$ the minimal geodesic in $\tilde{M}$ from $\tilde{a}$ to $\tilde{b}$ with respect to the induced metric. We have that the curves $p\left(\tilde{\gamma}_{\tilde{X}_{\tilde{t}_{k+1}}(\tilde{x}), \tilde{X}_{\tilde{t}_{k}}(\tilde{x})}\right)$ and $\gamma_{X_{t_{k}}\left(x_{k}\right), x_{k}}$ are homotopic, with endpoints fixed, since they both have the same endpoints and belong to a common ball in $M$. Consequently, the closed curves

$$
p \circ\left(\tilde{\beta}_{k}^{-1} * \tilde{\gamma}_{\tilde{X}_{\tilde{t}_{k+1}}(\tilde{x}), \tilde{X}_{\tilde{t}_{k}}(\tilde{x})} * \tilde{X}_{\left[\tilde{t}_{k}, \tilde{t}_{k+1}\right]}(\tilde{x}) * \tilde{\beta}_{k}\right)
$$

and

$$
\beta_{k}^{-1} * \gamma_{X_{t_{k}}\left(x_{k}\right), x_{k}} * X_{\left[0, t_{k}\right]}\left(x_{k}\right) * \beta_{k}
$$

are homotopic with the endpoint $x_{0}$ being fixed. From this we get

$$
\left[\beta_{k}^{-1} * \gamma_{X_{t_{k}}\left(x_{k}\right), x_{k}} * X_{\left[0, t_{k}\right]}\left(x_{k}\right) * \beta_{k}\right]_{x_{0}}=p_{\#}\left(\left[\tilde{\beta}_{k}^{-1} * \tilde{\gamma}_{\tilde{X}_{\tilde{t}_{k+1}}(\tilde{x}), \tilde{X}_{\tilde{t}_{k}}(\tilde{x})} * \tilde{X}_{\left[\tilde{t}_{k}, \tilde{t}_{k+1}\right]}(\tilde{x}) * \tilde{\beta}_{k}\right] \tilde{x}_{0}\right) \text {, }
$$

then

$$
\left[\beta_{k}^{-1} * \gamma_{X_{t_{k}}\left(x_{k}\right), x_{k}} * X_{\left[0, t_{k}\right]}\left(x_{k}\right) * \beta_{k}\right]_{x_{0}} \in p_{\#}\left(\pi_{1}\left(\tilde{M}, \tilde{x}_{0}\right)\right)=\operatorname{Ker}(\hat{u}),
$$

so

$$
u\left(\left[\beta_{k}^{-1} * \gamma_{X_{t_{k}}\left(x_{k}\right), x_{k}} * X_{\left[0, t_{k}\right]}\left(x_{k}\right) * \beta_{k}\right]\right)=0
$$

for all $k$ large. Since the closed paths $\beta_{k}^{-1} * \gamma_{X_{t_{k}}\left(x_{k}\right), x_{k}} * X_{\left[0, t_{k}\right]}\left(x_{k}\right) * \beta_{k}$ and $\gamma_{X_{t_{k}}\left(x_{k}\right), x_{k}} * X_{\left[0, t_{k}\right]}\left(x_{k}\right)$ are homologous (e.g. [15] p. 82) we conclude that

$$
u\left(\left[\gamma_{X_{t_{k}}\left(x_{k}\right), x_{k}} * X_{\left[0, t_{k}\right]}\left(x_{k}\right)\right]\right)=0
$$


for all $k$ large. It then follows from the definition of $\operatorname{def}(u)$ that every limit point of the set sequence $X_{\left[0, t_{k}\right]}\left(x_{k}\right)$ is contained in $\operatorname{def}(u)$. Since $M$ is compact we conclude that $\operatorname{def}(u) \neq \emptyset$.

On the other hand, $\operatorname{def}(u)$ consists of finitely many singularities since it is invariant (by Lemma 2.1) and finite (by hypothesis). Since every limit point of the sequence $X_{\left[0, t_{k}\right]}\left(x_{k}\right)$ lies in $\operatorname{def}(u)$ we conclude that there is $\sigma \in \operatorname{Sing}(X)$ such that

$$
\lim _{k \rightarrow \infty} \sup _{0 \leq t \leq t_{k}} \operatorname{dist}\left(X_{t}\left(x_{k}\right), \sigma\right)=0 .
$$

In particular, the length of the curves $X_{\left[0, t_{k}\right]}\left(x_{k}\right)$ go to 0 as $k \rightarrow \infty$. This would imply that the length of the corresponding curves $\tilde{X}_{\left[\tilde{t}_{k}, \tilde{t}_{k+1}\right]}(\tilde{x})$ go to 0 too, a contradiction since $y$ is not a singularity of $\tilde{X}$. This contradiction proves that every positive orbit of $\tilde{X}$ goes to either $\infty$ or $-\infty$ or a singularity. Analogously we prove that every negative orbit of $\tilde{X}$ goes to either $\infty,-\infty$ or a singularity.

To finish we prove that there are no positive (resp. negative) orbits going to $-\infty$ (resp. $\infty$ ). We shall prove it for the positive case since the negative one is similar. Again we proceed as in the proof of Theorem D in [5. Suppose by contradiction that there is $\tilde{x}$ such that $\tilde{X}_{t}(\tilde{x}) \rightarrow-\infty$. Take $x=p(\tilde{x})$, a limit point $y$ of $X_{t}(x)$ and $\tilde{y} \in p^{-1}(y)$. Then, there are sequences $n_{k} \in \mathbb{N}$ and $r_{k} \in \mathbb{R}$ such that $n_{k} \rightarrow \infty$, $r_{k} \rightarrow \infty$ and $d\left(\tilde{X}_{r_{k}}(\tilde{x}), g^{-n_{k}}(\tilde{y})\right) \rightarrow 0$. Consider the sequence of closed paths in $\tilde{M}$,

$$
\tilde{c}_{k}=\tilde{\delta}_{k} * \tilde{\gamma}_{\tilde{X}_{r_{k+1}}(\tilde{x}), g^{-n_{k+1}(\tilde{y})}} * \tilde{X}_{\left[r_{k}, r_{k+1}\right]}(\tilde{x}) * \tilde{\gamma}_{g^{-n_{k}}(\tilde{y}), \tilde{X}_{r_{k}}(\tilde{x})},
$$

where $\tilde{\delta}$ is a path from $g^{-n_{k+1}}(\tilde{y})$ to $g^{-n_{k}}(\tilde{y})$ and, again, $\tilde{\gamma}$ denotes the minimal geodesic in $\tilde{M}$. Clearly the closed curve $c_{k}=p\left(\tilde{c}_{k}\right)$ is homologous to the product

$$
\delta_{k} *\left(\gamma_{X_{r_{k+1}}(x), X_{r_{k}}(x)} * X_{\left[r_{k}, r_{k+1}\right]}(x)\right),
$$

where $\delta_{k}=p\left(\tilde{\delta}_{k}\right)$ is a closed path from $y$ to itself. But since $c_{k}$ lifts $\tilde{c}_{k}$, which is a closed path, and $u\left(\left[\delta_{k}\right]\right)=n_{k+1}-n_{k}$, we get $u\left(\left[c_{k}\right]\right)=0$ and then

$$
u\left(\left[\gamma_{X_{r_{k+1}}(x), X_{r_{k}}(x)} * X_{\left[r_{k}, r_{k+1}\right]}(x)\right]\right)=n_{k}-n_{k+1} \leq 0 .
$$

Therefore every point of the sequence $X_{\left[r_{k}, r_{k+1}\right]}(x)$ lies in $\operatorname{def}(u)$. However, $\operatorname{def}(u)$ consists of finitely many singularities, so the length of the paths $X_{\left[r_{k}, r_{k+1}\right]}(x)$ goes to 0 . This implies that the length of the corresponding paths $\tilde{X}_{\left[r_{k}, r_{k+1}\right]}(\tilde{x})$ also goes to 0 , which contradicts $n_{k} \rightarrow \infty$. This contradiction yields the result.

Next we give a geometric meaning for the deficiency. Given a closed transverse submanifold $S$ we define

$$
\sigma_{S}=\left\{x \in M: X_{t}(x) \notin S, \forall t \in \mathbb{R}\right\} .
$$

Define the Poincaré dual of $S$ as the cohomology class $u_{S} \in H^{1}(M, \mathbb{Z})$ whose value $u_{S}([c])$ at $[c] \in H_{1}(M, \mathbb{Z})$ is zero (if $c \cap S=\emptyset$ ) or $\Sigma_{p \in c \cap S} \epsilon_{p}$, where $\epsilon_{p}=1$ or -1 depending on whether $c$ intersects $S$ at $p$ in the direction given by $X$ or not (otherwise).

Proposition 2.2. If $S$ is a closed transverse submanifold of $X$, then $\operatorname{def}\left(u_{S}\right) \subset \sigma_{S}$.

Proof. For simplicity we write $u=u_{S}$. Take sequences $x_{k} \in M$ and $t_{k} \rightarrow \infty$ such that $d\left(X_{t_{k}}\left(x_{k}\right), x_{k}\right) \rightarrow 0$ and

$$
u\left(\left[\gamma_{X_{t_{k}}\left(x_{k}\right), x_{k}} * X_{\left[0, t_{k}\right]}\left(x_{k}\right)\right]\right) \leq 0, \quad \forall k \text { large }
$$


Since $M$ is compact we can assume $x_{k}$ (and hence $X_{t_{k}}\left(x_{k}\right)$ ) converge to some point $x \in M$.

We claim that $x \in \sigma_{S}$. Indeed, suppose by contradiction that $x \notin \sigma_{S}$. Then, there is $t \in \mathbb{R}$ such that $X_{t}(x) \in S$. By replacing $x$ by $X_{t}(x)$ if necessary we can assume that $t=0$ so $x \in S$. As $S$ is transverse to $X$ we can select $\epsilon>0$ (say) such that $X_{-\epsilon}(x) \notin S$. As in the proof of Lemma 2.1. since $X_{-\epsilon}$ is a diffeomorphism isotopic to the identity which commutes with $X_{t_{k}}$, we have

$$
\left[\gamma_{X_{t_{k}}\left(X_{-\epsilon}\left(x_{k}\right)\right), X_{-\epsilon}\left(x_{k}\right)} * X_{\left[0, t_{k}\right]}\left(X_{-\epsilon}\left(x_{k}\right)\right)\right]=\left[\gamma_{X_{t_{k}}\left(x_{k}\right), x_{k}} * X_{\left[0, t_{k}\right]}\left(x_{k}\right)\right] .
$$

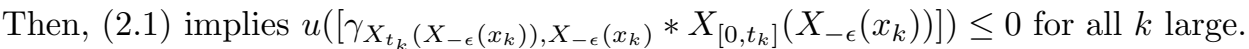
Since the positive orbits of $X$ intersects $S$ in the positive direction, we get

$$
X_{\left[0, t_{k}\right]}\left(X_{-\epsilon}\left(x_{k}\right)\right) \cap S=\emptyset .
$$

However, from the flow box theorem [13] applied to the positive orbit $X_{[0, \epsilon]}\left(X_{-\epsilon}(x)\right)$ we get

$$
X_{\left[0, t_{k}\right]}\left(X_{-\epsilon}\left(x_{k}\right)\right) \cap S \neq \emptyset
$$

since $X_{-\epsilon}\left(x_{k}\right) \rightarrow X_{-\epsilon}(x)$ and $t_{k} \rightarrow \infty$ as $k \rightarrow \infty$. This contradiction proves the claim.

Now we claim that

$$
\lim _{k \rightarrow \infty} \sup _{t \in\left[0, t_{k}\right]} d\left(X_{t}\left(x_{k}\right), \sigma_{S}\right)=0 .
$$

Indeed, if this is not true, then we can assume that there is a compact neighborhood $W$ of $\sigma_{S}$ with $W \cap S=\emptyset$ and $t_{k}^{\prime} \in\left[0, t_{k}\right]$ such that the sequence $X_{t_{k}^{\prime}}\left(x_{k}\right)$ converges to some $y \in \partial W$. We have that $t_{k}^{\prime}$ is not bounded because, otherwise, we would have that $y \in \sigma_{S}$ since $x_{k} \rightarrow x \in \sigma_{S}$ and $\sigma_{S}$ is an invariant set. Therefore $t_{k}^{\prime} \rightarrow \infty$. From this we get at once that $X_{t}(y) \notin S$ for all $t \geq 0$. On the other hand, if there were $t \geq 0$ such that $X_{-t}(y) \in S$, we would also have $t_{k}^{\prime \prime} \in\left[0, t_{k}^{\prime}\right]$ such that $X_{t_{k}^{\prime \prime}}\left(x_{k}\right) \in S$, again because $t_{k}^{\prime} \rightarrow \infty$. This is a contradiction which shows $y \in \sigma_{S}$. Since $W$ is a compact neighborhood of $\sigma_{S}$ and $y \in \partial W$, we get the contradiction proving (2.2). Now the result follows from (2.1), (2.2) and the definition of deficiency.

Next we give a simple characterization of the sphere $S^{n}, n \geq 2$, in terms of closed transverse submanifolds for vector fields (this characterization is clearly false for $n=1)$.

Lemma 2.3. A closed n-manifold $M$ is homeomorphic to $S^{n}$ if and only if there is a vector field $X$ all of whose singularities are hyperbolic in $M$ which exhibits a closed transverse submanifold $S$ intersecting all regular orbits such that $u_{S}=0$.

Proof. The only if part is trivial, so we only need to prove the if part. Suppose that $M$ exhibits a vector field $X$ with hyperbolic singularities and a closed transverse submanifold $S$ intersecting all regular orbits such that $u_{S}=0$. Given a singularity $\sigma$ we define its stable and unstable manifolds:

$$
W^{s}(\sigma)=\left\{x \in M: \lim _{t \rightarrow \infty} X_{t}(x)=\sigma\right\}, \quad W^{u}(\sigma)=\left\{x \in M: \lim _{t \rightarrow-\infty} X_{t}(x)=\sigma\right\} .
$$

It is well known that they are in fact immersed submanifolds of $M(7)$. We call $\sigma$ attracting or repelling depending on whether $\operatorname{dim}\left(W^{s}(\sigma)\right)=n$ or $\operatorname{dim}\left(W^{u}(\sigma)\right)=n$. Otherwise we call it saddle-type. For simplicity we write $u=u_{S}$. Notice that if 
there is $t>0$ such that $X_{t}(S) \cap S \neq \emptyset$, we could find a closed transverse path $c$ intersecting $S$ only once; thus $u([c]) \neq 0$, yielding $u \neq 0$, which is a contradiction. Therefore $X_{t}(S) \cap S=\emptyset$ for all $t>0$. From this we get the disjoint union

$$
S=\bigcup_{\sigma \in \operatorname{Sing}(X)} S_{\sigma}^{s},
$$

where $\operatorname{Sing}(X)$ denotes the set of singularities of $X$ and

$$
S_{\sigma}^{s}=\left\{x \in S \cap W^{s}(\sigma): X_{t}(x) \notin S, \forall t>0\right\} .
$$

Clearly if $\sigma$ is attracting, then $S_{\sigma}$ is an open subset of $S$. Let $x_{k} \in S_{\sigma}$ be a sequence converging to some $x \in S$. We have $x \in S_{\sigma^{\prime}}$ for some $\sigma^{\prime} \in \operatorname{Sing}(X)$ which must be saddle-type. Since $S$ intersects every regular orbit we have that the positive orbit of every point in $W^{u}\left(\sigma^{\prime}\right) \backslash\left\{\sigma^{\prime}\right\}$ intersects $S$. Now, take a closed path $c=\rho * \gamma * \beta * \alpha$ formed by a path $\alpha$ in the positive orbit of $x$ with $\alpha(1)$ being close to $\sigma^{\prime}$, a path $\beta$ from $\alpha(1)$ to some point $\beta(1) \in W^{s}\left(\sigma^{\prime}\right) \backslash\left\{\sigma^{\prime}\right\}$ close to $\sigma^{\prime}$, a path $\gamma$ in the positive orbit of $\beta(1)$ with $\gamma(1)$ close to $S$ and a path $\rho$ intersecting $S$ once. We can take $\alpha, \beta$ and $\gamma$ disjoint from $S$; thus $u([c]) \neq 0$ and so $u \neq 0$, a contradiction. This contradiction shows that $\sigma=\sigma^{\prime}$ and then $S_{\sigma}^{s}$ is a closed subset of $S$. Since $S$ is connected we conclude that $S=S_{\sigma}^{s}$. Analogously if we define

$$
S_{\sigma}^{u}=\left\{x \in S \cap W^{u}(\sigma): X_{t}(x) \notin S, \forall t<0\right\},
$$

then there is a repelling singularity $\sigma^{*}$ such that $S=S_{\sigma^{*}}^{u}$. Since $\sigma^{\prime}$ and $\sigma^{*}$ are attracting and repelling respectively we have that $S=S_{\sigma^{\prime}}^{s}=S_{\sigma^{*}}^{u}$ is the boundary of two $n$-balls $B^{\prime}$ and $B^{*}$, centered at $\sigma^{\prime}$ and $\sigma^{*}$ respectively, such that $M=B^{\prime} \cup B^{*}$. Therefore $M$ is diffeomorphic to $S^{n}$ (see for instance Theorem 6, p. 35, in [14]) and the result follows.

Proof of Theorem 1.3. Let $X$ be a vector field all of whose singularities are hyperbolic on a closed $n$-manifold $M \neq S^{n}, n \geq 2$. Consider a closed transverse submanifold $S$ intersecting all regular orbits. Since $M$ is closed we have that $X$ has finitely many singularities; hence $\sigma_{S}$ is finite. Therefore $\operatorname{def}\left(u_{S}\right)$ is finite by Proposition 2.2. Finally, $u_{S} \neq 0$ by Lemma 2.3 since $M \neq S^{n}$.

To finish we present two examples related to the above results. The first one is motivated by the fact that every cohomology class with finite deficiency is positive at every nonzero homology direction. Indeed, this fact made us ask if the conclusion of Theorem 1.2 holds for cohomology classes which are positive at every nonzero homology direction instead of the nonzero ones with finite deficiency. However, we have the following counterexample.

Example 2.4. There is a vector field in the 2-torus which is not gradient-like up to infinite cyclic coverings but exhibits a nonzero cohomology class which is positive at every nonzero homology direction.

This counterexample is described in Figure 1 $(u$ is nothing but the Poincaré dual of the transverse circle $S$ ). This example also shows that a cohomology class may have infinite deficiency even if it is positive at every nonzero homology direction.

The second example is motivated by the fact that there is a trivial example where the hypotheses of Corollary 1.1 are fulfilled: the suspended one. This observation made us ask if there are nontrivial examples where this corollary applies, namely, ones with singularities (all hyperbolic) on closed $n$-manifolds $\neq S^{n}, n \geq 2$, 


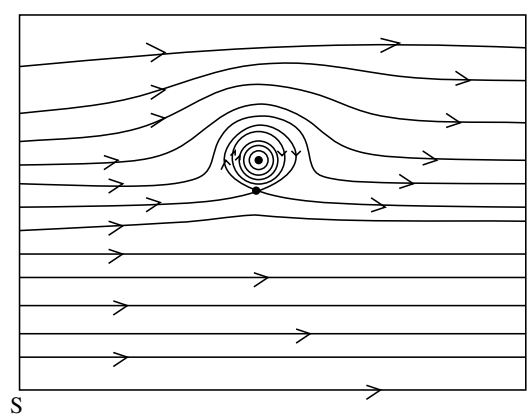

FiguRe 1

exhibiting closed transverse submanifolds intersecting all regular orbits. It is worth noting that the answer is negative for $n \in\{1,2\}$ and also for $n=3$ as soon as the vector field has only one singularity (see the proof of Proposition 3.9, p. 740, in [8]). Actually we have a positive answer for $n=3$ by the following:

Example 2.5. There is a closed 3-manifold $\neq S^{3}$ exhibiting a vector field with just two singularities (both hyperbolic) and a transverse torus intersecting all regular orbits.

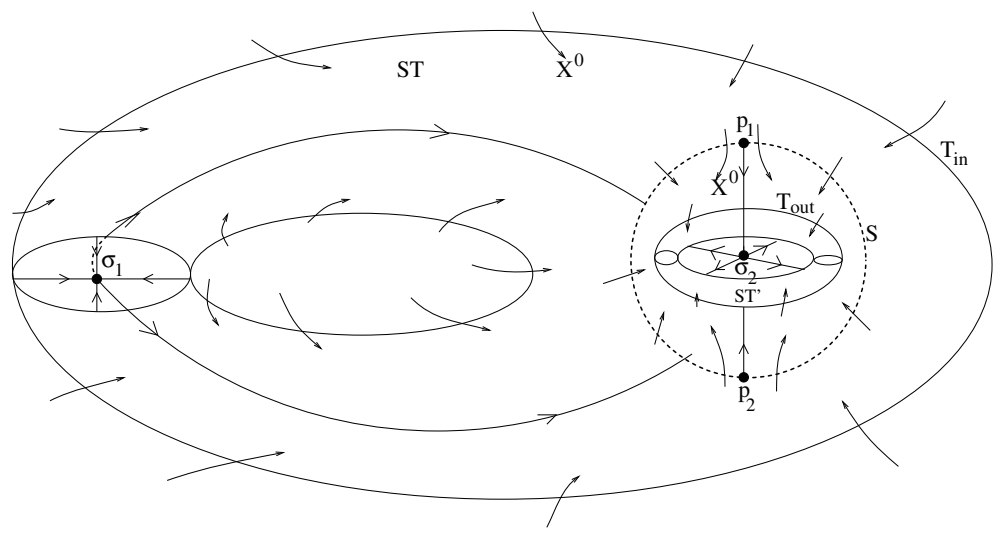

FigURE 2

Indeed, consider the solid tori $S T$ and $S T^{\prime} \subset \operatorname{Int}(S T)$ together with the vector field $X^{0}$ defined in the compact manifold $M^{0}=S T \backslash \operatorname{Int}\left(S T^{\prime}\right)$ depicted in Figure2, Observe that the boundary $\partial M^{0}$ of $M^{0}$ consists of two tori $T_{i n}=\partial S T$ (where $X^{0}$ points inward) and $T_{\text {out }}=\partial S T^{\prime}$ (where $X^{0}$ points outward). Notice also that $S T^{\prime}$ belongs to the interior of a 2-sphere $S$ where $X^{0}$ points inward. Gluing $T_{i n}$ to $T_{\text {out }}$ in a suitable way respecting the flow of $X^{0}$ in a way such that the two branches of the unstable manifold of $\sigma_{1}$ enter inside $S$ through points different from $p_{1}, p_{2}$, we obtain a closed manifold $M$ and a vector field $X$ with hyperbolic singularities $\left(\sigma_{1}, \sigma_{2}\right.$, say) on $M$ such that the common torus $T \approx T_{\text {in }} \approx T_{\text {out }}$ is a closed transversal intersecting all regular orbits. Evidently $M \neq S^{3}$ since $M$ has a nonseparating torus ( $T$ say). This ends the construction of the example. 


\section{ACKNowledgments}

The author would like to thank Professor A. Arbieto for helpful conversations and his students in the graduate course Anosov Flows, given in the second semester of 2009 at the Federal University of Rio de Janeiro, Brazil.

\section{REFERENCES}

1. Andrade, P., On homology directions for flows, Japan. J. Math. (N.S.) 17 (1991), no. 1, 1-9. MR 1115590 (92e:58183)

2. Basener, W., Every nonsingular flow on a closed manifold of dimension greater than two has a global transverse disk, Topology Appl. 135 (2004), no. 1-3, 131-148. MR2024952 (2004m:57047)

3. Basener, W., Transverse disks, symbolic dynamics, homology direction vectors, and ThurstonNielson theory, Topology Appl. 153 (2006), no. 14, 2760-2764. MR.2243748 (2007j:37031)

4. Collier, D., Sharp, R., Directions and equidistribution in homology for periodic orbits, Ergodic Theory Dynam. Systems 27 (2007), no. 2, 405-415. MR2308138(2008b:37041)

5. Fried, D., The geometry of cross sections to flows, Topology 21 (1982), no. 4, 353-371. MR670741 (84d:58068)

6. Fried, D., Flow equivalence, hyperbolic systems and a new zeta function for flows, Comment. Math. Helv. 57 (1982), no. 2, 237-259. MR684116 (84g:58083)

7. Hirsch, M., Pugh, C., Shub, M., Invariant manifolds. Lec. Notes in Math., 583. SpringerVerlag, Berlin-New York, 1977. MR0501173 (58:18595)

8. Morales, C. A., Axiom A flows with a transverse torus, Trans. Amer. Math. Soc. 355 (2003), no. 2, 735-745. MR1932723 (2003g:37049)

9. Mosher, L., Equivariant spectral decomposition for flows with a $\mathbb{Z}$-action, Ergodic Theory Dynam. Systems 9 (1989), no. 2, 329-378. MR.1007414 (90j:58117)

10. Mosher, L., Correction to: "Equivariant spectral decomposition for flows with a Z-action" [Ergodic Theory Dynamical Systems 9 (1989), no. 2, 329-378; MFҢ1007414||(90j:58117)], Ergodic Theory Dynam. Systems 10 (1990), no. 4, 787-791. MR.1091427 (94h:58155)

11. Mosher, L., Dynamical systems and the homology norm of a 3-manifold. I. Efficient intersection of surfaces and flows, Duke Math. J. 65 (1992), no. 3, 449-500. MR1154179 (93g:57018a)

12. Mosher, L., Dynamical systems and the homology norm of a 3-manifold. II, Invent. Math. 107 (1992), no. 2, 243-281. MR1144424 (93g:57018b)

13. Palis, J., Jr., de Melo, W., Geometric theory of dynamical systems. An introduction. Translated from the Portuguese by A. K. Manning. Springer-Verlag, New York-Berlin, 1982. MR669541 (84a:58004)

14. Rolfsen, D., Knots and links. Mathematics Lecture Series, 7. Publish or Perish, Inc., Houston, TX, 1990. MR.1277811 (95c:57018)

15. Rotman, J., An introduction to algebraic topology. Graduate Texts in Mathematics, 119. Springer-Verlag, New York, 1988. MR957919 (90e:55001)

Instituto de Matemática, Universidade Federal do Rio de Janeiro, P. O. Box 68530, 21945-970 Rio DE JANEIRo, BRAZIL

E-mail address: morales@impa.br 